\title{
Neuropsychobiology 1977;3:258-259
}

\section{Subject Index}

Affective disorders 199

Age distribution 144

Amanitin 1

Annual rhythm 35

Application 120

ATPases 129

Autokinetic response tendency 193

Avoidance, active 1

- learning 135

-, passive

Biochemical typology of depression 56 Blood/w vitro 120 Brain 129

- 5-HIAA 35

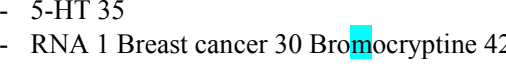

Carrier detection 144 Catecholamines 199 Catechol-O-methylttransferase 105 Central nervous system 144 Cesium 129 Circadian rhythm 35 Classification 56 Clomipramine 56 Cluster analysis 111 Contingent negative variation 12 CSF 5 HİAA 56 CyclicAMP 250

Depressed patient 49 Depression 56, 105,111, 167, 250 -, endogenous 23 Diagnostic in, dicators 167 Differential housing in mice 35

EEGsleep 167 EGOcloseness 193

- distance 193

Electrolytes 30,153 
Ethanol 135 Evoked potential 75 Extraversion 12

Follicle-stimulating hormone 160

Genetic counseling 144 Genetics 179

Hand 144

Heroin addiction 160 Heterozygote advantage 179 Highrisk 144 Huntington's chorea 144 5-Hydroxytryptamine 234

Indoleamines 199 Infantile autism 234

Laterality 144 £-Dopa 199

L-5-Hydroxy tryptophan 199 Lithium 30,129,153 /--Tryptophan 199 Luteinizing hormone 160

Manic-depressive patients 30,

153 MAO 65

Medical disease 167 Memory consolidation 1 Mental alertness 240 Methylphenidate 250 MHPG 23 - excretion 49 MIIA 65 Mice 120

Monoamine oxidase 105 Monoamines 199 Motion sickness 193 Motor decision 240

Neurophysiological variables 65 Neuropsychological testịng 167

Subject Index

Neuroticism 12 Nortriptyline 56

Odyssean personality 179

Paranoid 179

Parkinson's disease 42

Personality disorders 160

Pharmacopsychiatry 75

Phenelzine 49

Phenylalanine 199

Pituitary hormones 42

Plaitary hormones 42

Plasma aldosterone 153

Predictor of outcome

Premenstrual 105
Prolactin 30

Prolactin 30
Prolonged isolation 135

Proteịn kinases 129

Psychotic and high-risk children 75

Rats 120 Reaction time 12 Receptors 250 Rheoencephalography 240 Rubidium 120, 129 
Schizoid 179 Schizophrenịa 160

- in chilldhood 234 Schizophrenics 75 Serotonin 56,199

uptake 234 Sex difference 193 Sleep deprivation 23 Slow brain potentials 12 Strains of mice 135 Survival 179 Susceptibility 193

Testosterone 160 Thrombocytes 132 Thumb oppositịion 144 Tịıssues 120 Tyrosine 199

VAER 65

Voluntary movements 240

WPV 65 